\title{
The white dwarf binary pathways survey - II. Radial velocities of 1453 FGK stars with white dwarf companions from LAMOST DR 4
}

\author{
A. Rebassa-Mansergas, ${ }^{1,2 \star ~ J . ~ J . ~ R e n, ~}{ }^{3}$ P. Irawati, ${ }^{4}$ E. García-Berro, ${ }^{1,2}$ S. G. Parsons, ${ }^{5}$ \\ M. R. Schreiber, ${ }^{6}$ B. T. Gänsicke, ${ }^{7}$ P. Rodríguez-Gil, ${ }^{8,9}$ X. Liu, ${ }^{10}$ C. Manser, ${ }^{7}$ \\ S. P. Nevado, ${ }^{8,9}$ F. Jiménez-Ibarra, ${ }^{8,9}$ R. Costero, ${ }^{11}$ J. Echevarría, ${ }^{11}$ \\ R. Michel, ${ }^{12}$ M. Zorotovic, ${ }^{6}$ M. Hollands, ${ }^{7}$ Z. Han, ${ }^{13}$ A. Luo, ${ }^{3}$ E. Villaver ${ }^{14}$ \\ and X. Kong ${ }^{3}$ \\ ${ }^{1}$ Departament de Física, Universitat Politècnica de Catalunya, c/Esteve Terrades 5, E-08860 Castelldefels, Spain \\ ${ }^{2}$ Institute for Space Studies of Catalonia, c/Gran Capità 2-4, Edif. Nexus 201, E-08034 Barcelona, Spain \\ ${ }^{3}$ National Astronomical Observatories, Chinese Academy of Sciences, 100012 Beijing, P. R. China \\ ${ }^{4}$ National Astronomical Research Institute of Thailand, Sirindhorn AstroPark, Donkaew, Mae Rim, Chiang Mai 50180, Thailand \\ ${ }^{5}$ Deparment of Physics \& Astronomy, University of Sheffield, Sheffield S3 7RH, UK \\ ${ }^{6}$ Instituto de Física y Astronomía, Universidad de Valparaíso, Avenida Gran Bretaña 1111, Valparaíso, Chile \\ ${ }^{7}$ Department of Physics, University of Warwick, Coventry CV4 7AL, UK \\ ${ }^{8}$ Instituto de Astrofísica de Canarias, c/ Vía Láctea s/n, E-38205 La Laguna, Tenerife, Spain \\ ${ }^{9}$ Universidad de La Laguna, Departamento de Astrofísica, E-38206 La Laguna, Tenerife, Spain \\ ${ }^{10}$ Department of Astronomy, Peking University, Beijing 100871, P. R. China \\ ${ }^{11}$ Instituto de Astronomía, Universidad Nacional Autónoma de México, 70-264, Ciudad Universitaria, México D.F., C.P. 04510, México \\ ${ }^{12}$ Instituto de Astronomía, Universidad Nacional Autónoma de México, 877, Ensenada, Baja California, C.P. 22830 México \\ ${ }^{13}$ Key Laboratory for the Structure and Evolution of Celestial Objects, Yunnan observatories, Chinese Academy of Sciences, PO Box 110, Kunming, 650011, \\ Yunnan Province, P. R. China \\ ${ }^{14}$ Departamento de Física Teórica, Universidad Autónoma de Madrid, Cantoblanco E-28049 Madrid, Spain
}

\begin{abstract}
We present the second paper of a series of publications aiming at obtaining a better understanding regarding the nature of type Ia supernovae (SN Ia) progenitors by studying a large sample of detached F, G and $\mathrm{K}$ main-sequence stars in close orbits with white dwarf companions (i.e. WD+FGK binaries). We employ the Large Sky Area Multi-Object Fibre Spectroscopic Telescope (LAMOST) data release 4 spectroscopic data base together with Galaxy Evolution Explorer (GALEX) ultraviolet fluxes to identify 1549 WD+FGK binary candidates (1057 of which are new), thus doubling the number of known sources. We measure the radial velocities of 1453 of these binaries from the available LAMOST spectra and/or from spectra obtained by us at a wide variety of different telescopes around the globe. The analysis of the radial velocity data allows us to identify 24 systems displaying more than $3 \sigma$ radial velocity variation that we classify as close binaries. We also discuss the fraction of close binaries among WD+FGK systems, which we find to be $\sim 10$ per cent, and demonstrate that high-resolution spectroscopy is required to efficiently identify double-degenerate SN Ia progenitor candidates.
\end{abstract}

Key words: stars: low-mass - white dwarfs - binaries (including multiple): close.

\section{INTRODUCTION}

Thermonuclear supernovae, also known as Type Ia supernovae ( $\mathrm{SN}$ Ia), originate from the explosion of carbon-oxygen white dwarfs

^E-mail: alberto.rebassa@upc.edu
(WDs; Hoyle \& Fowler 1960), and are among the most luminous events in the Universe. Consequently, SN Ia can be detected up to very large distances. Since they arise from the detonation of WDs in a narrow mass range, they have nearly the same intrinsic luminosity, and thus they play a primary role as standardizable candles in cosmology. In particular, the discovery of a relation between their intrinsic luminosity and the shape of their light curves (Phillips 1993) 
has paved the way to a new era of precision cosmology, leading to the discovery of the acceleration of the Universe (Riess et al. 1998; Schmidt et al. 1998; Perlmutter et al. 1999; Astier \& Pain 2012). However, although SN Ia observations have led to fundamental discoveries, the nature of their progenitors is not yet unambiguously determined. Even more, albeit their intrinsic importance, the explosion mechanism of SN Ia still remains poorly understood after more than five decades of theoretical and observational work, thus compromising their use and possibly introducing some not yet well-known systematic errors in the determination of extragalactic distances (Linden, Virey \& Tilquin 2009; Howell 2011).

It is widely accepted that a WD in a binary system is at the heart of an SN Ia outburst. Several possible evolutionary channels leading to such an event are currently envisaged, all of which have advantages and drawbacks. Hence, there is not yet a general consensus on the leading path for SN Ia. In fact, it is well possible that all of them contribute to the total rate in some unknown fraction (Wang $\&$ Han 2012). These evolutionary channels are briefly described below.

In the single degenerate channel (Whelan \& Iben 1973; Nomoto 1982; Han \& Podsiadlowski 2004), the WD accretes mass from a non-degenerate stellar donor and explodes when its mass grows near the Chandrasekhar limit. In the double-detonation mechanism (Woosley \& Weaver 1994; Livne \& Arnett 1995) a WD with a mass smaller than the Chandrasekhar limit accumulates heliumrich material on its surface from its companion. The helium layer is compressed as more material is accreted and ultimately detonates. The compression wave propagates towards the centre and a second detonation occurs near the centre of the carbon-oxygen core - see, for instance, Shen et al. (2012) and references therein. Next comes the double-degenerate channel (Whelan \& Iben 1973; Nomoto 1982; Iben \& Tutukov 1984). In this scenario, two WDs merge and a prompt violent detonation ensues when the masses of the two merging WDs are sufficiently large, otherwise a delayed explosion could eventually occur. Recently, another possible scenario has been proposed, the so-called core-degenerate channel (Livio \& Riess 2003; Kashi \& Soker 2011; Soker et al. 2013), where a WD merges with the hot core of a massive asymptotic giant branch (AGB) star during or after a common envelope phase. Finally, another possible channel involves the collision of two WDs in a dense stellar environment (Katz, Dong \& Malhotra 2011; Thompson 2011; Aznar-Siguán et al. 2013; Kushnir et al. 2013). In this scenario, either a tertiary star brings two WDs to collide or the dynamical interaction occurs in a dense stellar system, where such interactions are likely. It has been shown that, in some cases, the collision results in an immediate explosion. However, this scenario can only account for a few per cent of all SN Ia under the most favourable assumptions (Hamers et al. 2013; Soker et al. 2014). In summary, the challenging, unsolved problem of understanding and quantifying the contribution of the different progenitor channels to the observed population of SN Ia is still a matter of active research.

Currently, the two most popular scenarios for SN Ia are the singleand the double-degenerate channels. The viability of both paths has been intensively studied during the last several years from a theoretical perspective (e.g. Hachisu et al. 2012; Bours, Toonen \& Nelemans 2013; Nielsen et al. 2014; Jiménez, Tissera \& Matteucci 2015; van Rossum et al. 2016; Yungelson \& Kuranov 2017). Unfortunately, these theoretical studies have yielded so far no conclusive results on which of these two channels is more efficient in producing SN Iae. For instance, the mass growth of the WD in the singledegenerate channel is thought to be rather small (e.g. Idan, Shaviv \& Shaviv 2012) avoiding reaching a mass near the Chandrasekhar limit. Moreover, determinations of the SN Ia delay time distributions are not consistent with expectations for single-degenerate systems (Gal-Yam \& Maoz 2004; Totani et al. 2008). In contrast, the double-degenerate channel predicts a delay time distribution in better agreement with observations (Maoz \& Badenes 2010; Maoz, Sharon \& Gal-Yam 2010). However, current simulations predict that the range of WD masses that produce powerful detonations is rather limited, and in most of the cases the outcome of the merger could result in other (interesting) astrophysical phenomena. Specifically, it has been found that a possible outcome could be an accretion-induced collapse to a neutron star (Nomoto \& Iben 1985; Shen et al. 2012), whereas in some other cases a high-field magnetic WD could be formed (García-Berro et al. 2012). Observationally, several additional analyses have provided some support for both the single- (Hamuy et al. 2003; Livio \& Riess 2003; Voss \& Nelemans 2008; Liu \& Stancliffe 2016; Shappee et al. 2016) and the double-degenerate channels (González Hernández et al. 2012; Olling et al. 2015; Santander-García et al. 2015). However, there is no single system yet that has robustly been confirmed as a singleor double-degenerate SN Ia progenitor - see e.g. García-Berro et al. (2016).

With these thoughts in mind, we have started an observational campaign that aims at providing additional observational input for testing both the single- and double-degenerate channels for SN Ia based on observations of WDs in detached close binaries where the secondary star is an F, G or K main-sequence star (hereafter WD+FGK binaries). These systems were initially main sequence binaries that evolved through common envelope evolution and have now orbital periods ranging from a few hours to several weeks (Willems \& Kolb 2004). Depending on the orbital periods and component masses, the WD+FGK binaries are expected to evolve mainly through the following two channels. In the first path, the secondary star transfers mass to the WD in a thermal time-scale, ensuing the so-called super-soft source (SSS) phase. The mass transfer rate is high enough to sustain stable hydrogen burning so the WD mass grows. Hence, the system becomes a potential singledegenerate SN Ia progenitor (Shara, Prialnik \& Shaviv 1977; Fujimoto 1982; Iben 1982; van den Heuvel et al. 1992; Di Stefano 2010; Wolf et al. 2013). If the WD does not accrete sufficient mass during the SSS phase the WD+FGK becomes a cataclysmic variable with a massive WD and an evolved donor (e.g. Schenker et al. 2002; Thorstensen et al. 2002; Gänsicke et al. 2003; Zorotovic, Schreiber \& Gänsicke 2011; Rebassa-Mansergas et al. 2014). Another alternative is that the system may go through a second common envelope phase. In this case, the envelope may be ejected and the result is a double WD in a tight orbit, i.e. a potential double-degenerate SN Ia progenitor. Depending on the time that it takes to bring the two WDs close enough to overflow the Roche lobe (it should be shorter than the Hubble time) and the total mass of the system (it should be close to the Chandrasekhar mass) the result could be an SN Ia outburst. By analysing a large sample of close WD+FGK binaries with well-determined orbital periods and component masses, we will be able to reconstruct their past evolution, and to predict their future evolution and thus quantify the fraction of systems evolving through each path. It is important to keep in mind that our aim here is not to identify individual SN Ia progenitors (in fact the vast majority of our close WD+FGK binaries will not become SN Ia), but to test our understanding of the binary evolution theory of the single- and double-degenerate channels towards SN Ia.

In Parsons et al. (2016), we presented the first of a series of publications resulting from our dedicated campaign, where we described our methodology to efficiently identify WD+FGK binaries 


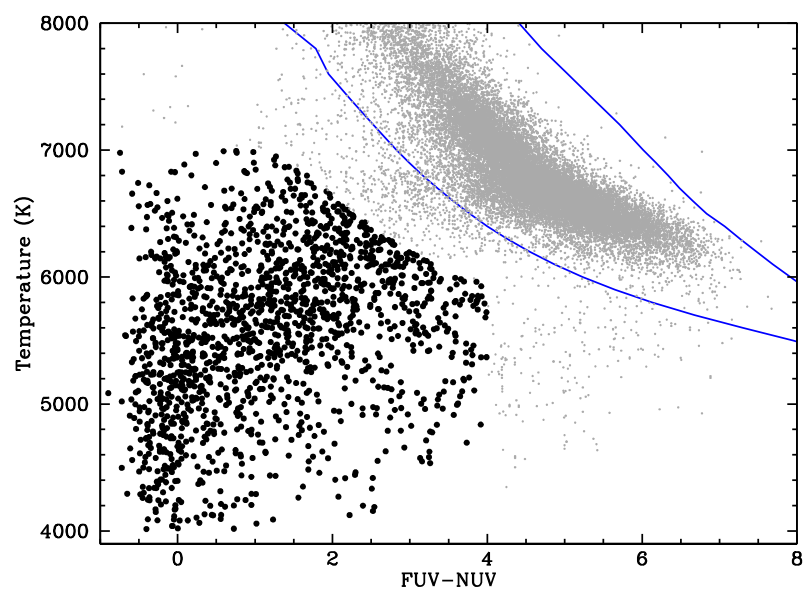

Figure 1. The UV colours and temperatures of LAMOST main-sequence FGK stars (grey dots). The temperatures are taken directly from the LAMOST DR 4 catalogue. Shown in blue are two theoretical tracks computed from PHOENIX atmospheric models, one with a high metallicity and high surface gravity $(\log Z=+1$ and $\log g=5.0$, top curve) and one with a low metallicity and low surface gravity $(\log Z=-3$ and $\log g=3.5$, bottom curve). These represent extreme limits on the colours of main-sequence stars. The vast majority of stars fall within these extreme models, as expected. The black solid points mark the 1599 targets that fall within our cut for selecting WD+FGK binary candidates (FUV-NUV $<4,8000>T>4000 \mathrm{~K}$ and FUV-NUV at least 1.5 mag bluer than the bottom PHOENIX model).

(see Parsons et al. 2015a for our study on the first SSS progenitor identified so far). These were selected as F, G, K stars with Northern hemisphere Large Sky Area Multi-Object Fibre Spectroscopic Telescope (LAMOST; Cui et al. 2012; Zhao et al. 2012) or southern-hemiesphere Radial Velocity Experiment (RAVE; Kordopatis et al. 2013) spectra displaying ultraviolet (UV) excess as indicated by their Galaxy Evolution Explorer (GALEX; Martin et al. 2005) FUV-NUV colours (where FUV and NUV are the farUV and near-UV magnitudes) in a $T_{\text {eff }}$ versus FUV-NUV diagram (see Fig. 1). This resulted in $430 \mathrm{WD}+\mathrm{FGK}$ binary candidates from RAVE DR 1 and 504 candidates from LAMOST DR 1.

In this paper, we first expand our WD+FGK binary sample by applying our selection criteria to the FGK star catalogue from LAMOST DR 4. We then use LAMOST and follow-up spectroscopic observations of $1453 \mathrm{WD}+\mathrm{FGK}$ binary candidates in the new LAMOST sample for measuring their radial velocities and we identify 24 close binaries displaying significant radial velocity variations. Finally, we discuss the close binary fraction among WD+FGK binaries. In a forthcoming publication we will present a radial velocity analysis dedicated to follow-up our Southern hemisphere RAVE targets as well as our first orbital period measurements.

\section{THE DR 4 OF LAMOST}

LAMOST is $\mathrm{a} \simeq 4 \mathrm{~m}$ quasi-meridian reflecting Schmidt telescope located at Xinglong Observing Station in the Hebei province of China (Cui et al. 2012). It has a field of view of $5^{\circ}$ in diameter and it is exclusively dedicated to obtain optical spectroscopy of celestial objects. Each 'spectral plate' refers physically to a focal surface with 4000 precisely positioned optical fibers to observe spectroscopic plus calibration targets simultaneously, equally distributed among 16 fibre-fed spectrographs. Each spectrograph is equipped with two CCD cameras of blue and red channels that simultaneously provide blue and red spectra of the 4000 selected targets, respectively. The spectra cover the $\sim 3700-9000 \AA$ wavelength range at a resolving power of $\sim 1800$.

Since 2012 September LAMOST has been performing a 5-yr regular survey, which was preceded by a 2 -yr commissioning survey and a 1-yr pilot survey. The LAMOST regular survey consists of two main parts (Zhao et al. 2012): the LAMOST Extra-Galactic Survey (LEGAS) of galaxies to study the large-scale structure of the Universe, and the LAMOST Experiment for Galactic Understanding and Exploration (LEGUE) Survey of the Milky Way which is developed to obtain millions of stellar spectra to study the structure and evolution of the Galaxy (Deng et al. 2012). LEGUE is subdivided into three independent surveys for follow-up observations of the spheroid, the disc and the galactic anticentre, respectively (Carlin et al. 2012; Chen et al. 2012; Yuan et al. 2015; Xiang et al. 2017a,b).

The raw spectra are reduced by the LAMOST two-dimensional (2D) pipeline (Luo et al. 2012, 2015), which extracts spectra from raw CCD images and calibrates them. This procedure includes dark and bias subtraction, cosmic ray removal, one-dimensional (1D) spectral extraction, flat-field correction, wavelength calibration, sky subtraction, merging sub-exposures and combining wavelength bands (after flux calibration). Each LAMOST spectrum is the result of combining a certain number of individual sub-exposures $(\geq 3)$, and in several cases the targets are observed in different nights. In a second step, the 1D pipeline works on spectral type classification (four primary types: star, galaxy, quasar, unknown) and radial velocity (or redshift) measurement for galaxies and quasars. Finally, the LAMOST Stellar Parameter Pipeline (LASP) accurately and automatically determines the fundamental stellar parameters for late A and FGK type stellar spectra (effective temperature $T_{\text {eff }}$, surface gravity: logg, metallicity $[\mathrm{Fe} / \mathrm{H}]$ and radial velocity $\mathrm{RV})$. To that end LASP adopts two consecutively methods - the Correlation Function Initial (CFI) value and Université de Lyon Spectroscopic analysis Software (ULYSs; Wu et al. 2011). The CFI method is used to produce a set of coarse measurements that serve as initial guesses for ULYSS, which then determines the final stellar parameters. By comparing with the estimates from high-resolution spectra, the $T_{\text {eff }}, \log g$ and $[\mathrm{Fe} / \mathrm{H}]$ determined by LASP have the external errors $-47 \pm 95 \mathrm{~K}$, $0.03 \pm 0.25 \mathrm{dex}$ and $-0.02 \pm 0.1 \mathrm{dex}$, respectively (Luo et al. 2015).

The latest data product of LAMOST is date release 4 (DR 4), currently only released internally to the Chinese astronomical community. ${ }^{1}$ DR 4 includes spectra observed from 2011 October 24 to 2016 June 2 by 3454 different plates. DR 4 thus contains a total of 7681185 spectra, of which 6898298 are catalogued as stars, 118743 as galaxies, 41352 as quasars and 622792 are unknown objects. Furthermore, in addition to the spectral data and the general catalogue mentioned above, LAMOST DR 4 also contains three catalogues including measured stellar parameters for (1) late A and FGK-type main-sequence stars with high quality spectra (4 202127 entries), (2) A-type main-sequence stars (364 600 entries) and (3) M-type main-sequence stars (433247 entries).

\section{WD+FGK BINARIES IN LAMOST DR 4}

WD+FGK binaries are not easy to identify in the optical since the main-sequence stars generally outshine the WDs (RebassaMansergas et al. 2010). Hence, UV coverage is required to detect the WD. As we have already mentioned, in Parsons et al. (2016) we

\footnotetext{
${ }^{1}$ http://dr4.lamost.org/
} 
Table 1. The selected 2168 spectra of 1549 unique LAMOST DR 4 WD+FGK binary candidates (see Fig. 1), including coordinates, GALEX UV magnitudes, spectral ID identifiers (plate, spectrograph, fibre IDs and modified Julian date MJD), signal-to-noise ratio in different spectral bands and stellar parameters. The 'Mag' column provides the optical magnitudes obtained from AAVSO Photometric All Sky Survey DR9 (APASS; Henden et al. 2015), although some entries are obtained from the Catalina Sky Survey (CSS; Drake et al. 2009) or Guiding Star Catalog (GSC). Only for few systems with no available $V$ magnitudes we report the $g$ magitude from the Sloan Digital Sky Survey (SDSS; York et al. 2000) or the Xuyi Schmidt Telescope Photometric Survey of the Galactic Anticentre (XSTPS-GAS; Liu et al. 2014; Zhang et al. 2014). The complete table can be found in the electronic version of the paper.

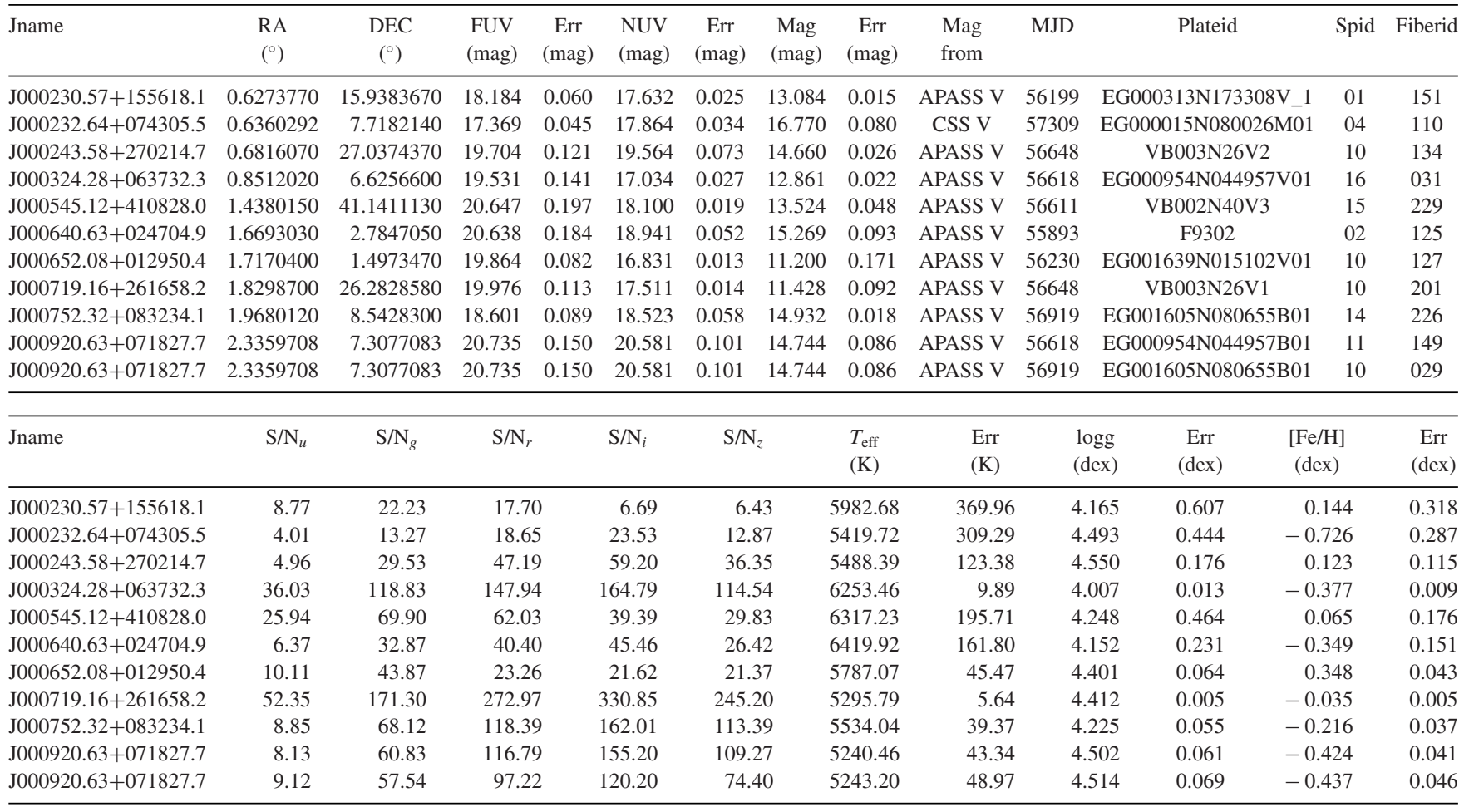

developed a method for efficiently identifying WD+FGK binaries based on the following criteria (also illustrated in Fig. 1):

$\mathrm{eFUV} \leq 0.2, \mathrm{eNUV} \leq 0.2,(\mathrm{FUV}-\mathrm{NUV}) \leq 4$,

$4000 \mathrm{~K} \leq T_{\text {eff }} \leq 7000 \mathrm{~K}, e T_{\text {eff }} \leq 500 \mathrm{~K}$,

$$
\begin{aligned}
T_{\text {eff }}< & 8592.91552-2747.51667 \times(\mathrm{FUV}-\mathrm{NUV}) \\
& +2292.51782 \times(\mathrm{FUV}-\mathrm{NUV})^{2}-1279.40096 \\
& \times(\mathrm{FUV}-\mathrm{NUV})^{3}+407.13454 \\
& \times(\mathrm{FUV}-\mathrm{NUV})^{4}-71.88852 \\
& \times(\mathrm{FUV}-\mathrm{NUV})^{5}+6.56399 \\
& \times(\mathrm{FUV}-\mathrm{NUV})^{6}-0.24160 \times(\mathrm{FUV}-\mathrm{NUV})^{7}
\end{aligned}
$$

NUV_art $=0$, FUV_art $=0$,

where eFUV and eNUV are the NUV and FUV magnitude errors, $T_{\text {eff }}$ is the effective temperature provided by the LAMOST DR 4 stellar parameter pipeline and NUV_art and FUV_art are the NUV and FUV artefacts, i.e. we choose only GALEX photometry associated with no artefacts. This results in 2226 spectra of 1599 unique main sequence FGK stars being selected as WD+FGK binary candidates (black solid dots in Fig. 1) among the 4202127 entries in the LAMOST DR 4 late A, FGK-type main sequence stellar parameter catalogue. Visual inspection of the 2226 selected spectra revealed the typical features of FGK stars in all cases except for seven objects associated with bad quality spectra that we decided to exclude. Moreover, we cross-correlated our list with the Simbad catalogue and found: nine eclipsing binaries of W UMa type, one eclipsing main sequence binary, one eclipsing binary of Lyr type, one eclipsing binary of Algol type, two classical cepheids, 26 variable stars of RR Lyr type, one symbiotic star, one red giant branch star, one T Tauri star and three RS CVn binaries. We excluded all these sources from our list except the three RS CVn binaries since, a priori, these systems may contain a WD and a chromospherically active mainsequence companion, e.g. V471 Tau (Hussain et al. 2006). ${ }^{2}$ This left us with a total number of 2168 spectra of 1549 unique WD+FGK binary candidates. This list includes the 504 LAMOST DR 1 objects we already presented in Parsons et al. (2016) except for 13 sources that were excluded by LAMOST due to pipeline updates. Table 1 includes all the 1549 objects and the corresponding duplicate spectra, where we also provide coordinates, stellar parameters as obtained from the DR 4 stellar parameters pipeline, GALEX and optical magnitudes, spectral ID identifiers, signal-to-noise ratio of the spectra and Simbad classification when available. In the following section, we present a follow-up radial velocity survey for identifying close binaries among the $1549 \mathrm{WD}+\mathrm{FGK}$ systems.

\section{OBSERVATIONS}

In the previous section, we have provided details on our strategy for efficiently identifying WD+FGK binaries. However, it is important to keep in mind that only a fraction of these systems $(\sim 1 / 4$; see

\footnotetext{
${ }^{2}$ Simbad lists 2, 16 and 6 of our objects as quasars, galaxies and globular clusters, respectively. However, we do not exclude these sources from our list as visual inspection of their LAMOST spectra clearly revealed they are FGK main-sequence stars
} 
Table 2. Log of the observations. We include here only follow-up spectroscopy obtained by our team. The MJD of all LAMOST DR 4 spectra used in this work can be found in Table 1. We indicate the telescopes and the spectrographs used, the resolving powers of the spectra, the type of observation (visitor or service), the date and the number of spectra obtained per night.

\begin{tabular}{|c|c|c|c|c|c|}
\hline Telesc. & Spectrograph & $R$ & Type & Date & \#Spectra \\
\hline Palomar 5.1 & DBSP & 6370 & Visitor & 2014 January 19 & 36 \\
\hline Palomar 5.1 & DBSP & 6370 & Visitor & 2014 January 21 & 9 \\
\hline CFHT & Espadons & 68000 & Service & 2015 August & 48 \\
\hline CFHT & Espadons & 68000 & Service & 2016 January & \\
\hline TNT & MRES & 15000 & Visitor & 2015 February 3 & 5 \\
\hline TNT & MRES & 15000 & Visitor & 2015 February 4 & 11 \\
\hline TNT & MRES & 15000 & Visitor & 2015 February 5 & 2 \\
\hline TNT & MRES & 15000 & Visitor & 2015 February 6 & 1 \\
\hline TNT & MRES & 15000 & Visitor & 2015 April 1 & 7 \\
\hline TNT & MRES & 15000 & Visitor & 2015 April 2 & 6 \\
\hline TNT & MRES & 15000 & Visitor & 2015 May 11 & 2 \\
\hline TNT & MRES & 15000 & Visitor & 2015 May 12 & 4 \\
\hline TNT & MRES & 15000 & Visitor & 2015 May 13 & 1 \\
\hline TNT & MRES & 15000 & Visitor & 2016 April 13 & 7 \\
\hline TNT & MRES & 15000 & Visitor & 2016 April 27 & 1 \\
\hline TNT & MRES & 15000 & Visitor & 2016 April 28 & 3 \\
\hline TNT & MRES & 15000 & Visitor & 2017 March 4 & 6 \\
\hline TNT & MRES & 15000 & Visitor & 2017 March 5 & 9 \\
\hline TNT & MRES & 15000 & Visitor & 2017 March 6 & 11 \\
\hline TNT & MRES & 15000 & Visitor & 2017 April 27 & 3 \\
\hline TNT & MRES & 15000 & Visitor & 2017 April 29 & 1 \\
\hline TNT & MRES & 15000 & Visitor & 2017 April 30 & 4 \\
\hline INT & IDS & 6600 & Visitor & 2016 January 23 & 15 \\
\hline INT & IDS & 6600 & Visitor & 2016 January 24 & 19 \\
\hline INT & IDS & 6600 & Visitor & 2016 January 25 & 26 \\
\hline INT & IDS & 6600 & Visitor & 2016 November 15 & 1 \\
\hline INT & IDS & 6600 & Visitor & 2016 November 16 & 20 \\
\hline INT & IDS & 6600 & Visitor & 2016 November 19 & 12 \\
\hline INT & IDS & 6600 & Visitor & 2016 November 20 & 8 \\
\hline INT & IDS & 6600 & Visitor & 2016 November 21 & 15 \\
\hline INT & IDS & 6600 & Service & 2016 November 18 & 9 \\
\hline INT & IDS & 6600 & Service & 2016 November 19 & 26 \\
\hline SPM 2.1 & Echelle & 20000 & Visitor & 2015 December 5 & 9 \\
\hline SPM 2.1 & Echelle & 20000 & Visitor & 2015 December 6 & 18 \\
\hline SPM 2.1 & Echelle & 20000 & Visitor & 2015 December 7 & 12 \\
\hline TNG & HARPS-N & 115000 & Visitor & 2017 January 1 & 25 \\
\hline TNG & HARPS-N & 115000 & Visitor & 2017 January 2 & 25 \\
\hline TNG & HARPS-N & 115000 & Visitor & 2017 January 3 & 24 \\
\hline TNG & HARPS-N & 115000 & Visitor & 2017 January 4 & 23 \\
\hline
\end{tabular}

e.g. Willems \& Kolb 2004) are expected to have evolved through mass-transfer interactions such as a common envelope phase and become close compact binaries of orbital periods ranging from a few hours to several weeks. In order to identify these close binaries, we require radial velocity information, which we obtained from the available LAMOST DR 4 spectra as well as from our own followup spectroscopy taken at several different telescopes around the globe (see a log of the observations in Table 2). In the following, we describe the spectroscopic observations.

\subsection{LAMOST DR 4}

As mentioned above, the current data product of LAMOST is DR 4, which includes 4202127 spectra of late A and FGK main-sequence stars with available stellar parameters. Among these we have selected 1549 as WD+FGK binary candidates (Section 3). The total number of combined available LAMOST spectra for our selected targets is 2168 , or 5357 if we take into account the individual subexposures of each combined spectrum. We also cross-correlated our list of LAMOST WD+FGK binaries with the spectroscopic data base of the Sloan Digital Sky Survey (SDSS; York et al. 2000) data release 12, which resulted in 88 additional spectra for 21 of our targets. The resolving power of both LAMOST and SDSS spectra is $R \sim 1800$.

\subsection{Palomar Hale Telescope}

Follow-up spectroscopy for $44 \mathrm{WD}+\mathrm{FGK}$ binaries was obtained during two nights from the 5.1-m Palomar Hale Telescope in 2014 January 19 and 21 (Table 2) at Palomar Observatory in north San Diego County, California, USA. Two different gratings were used, the 1200 and 600 lines $\mathrm{mm}^{-1}$, providing spectra of the red and blue arms, respectively. For this the double spectrograph (DBSP), together with a 1-arcsec width long-slit were used. Arc spectra were taken at the beginning of the nights and we used the sky lines to account the flexure of the instrument (note that we only made use of the red arm spectra to measure the radial velocities). The resolving power was 6370 in the red arm covering the $\sim 7600$ $9300 \AA$ wavelength range. The blue arm had a resolving power of 1380, ranging from 3500 to $6500 \AA$.

\subsection{Isaac Newton Telescope}

Eight nights in 2014 December, 2016 January and 2016 November plus $4 \mathrm{~h}$ of service mode observations in 2016 November (Table 2) at the the Issac Newton Telescope (INT) in Roque de los Muchachos Observatory at La Palma, Spain provided follow-up spectroscopy for $47 \mathrm{WD}+\mathrm{FGK}$ binaries. The spectra were obtained from the $1200 Y$ grating. A 1-arcsec wide long-slit together with the IDS (Intermediate Dispersion Spectrographs) were used throughout the whole observations. The spectra covered the $\sim 5100-6400$ $\AA$ A wavelength range at a resolving power of 6600 . One arc spectrum was taken at the beginning each night. We calibrated the spectra using the single arc spectrum and adjusted their calibration using the night sky emission lines.

\subsection{Canada-France-Hawaii Telescope}

The Canada-France-Hawaii Telescope (CFHT) provided follow-up spectroscopy for $25 \mathrm{WD}+\mathrm{FGK}$ binaries. The observations were carried in service mode from 2015 August to 2016 January (Table 2) at Mauna Kea Observatory, Hawaii, USA. For this a 1.6 arcsec aperture hole was used together with the Echelle SpectroPolarimetric Device for the Observation of Stars (Espadons) spectrograph. The spectra covered the entire optical wavelength range at a resolving power of 68000 . Arc spectra were obtained before each science spectrum.

\subsection{Thai National Telescope}

Additional follow-up spectroscopy for $30 \mathrm{WD}+\mathrm{FGK}$ binaries was obtained from the Thai National Telescope (TNT) at the That National Observatory in Thailand during 20 nights spread between 2015 and 2017 (see Table 2). Using a 1.4-arscsec slit width, the light was redirected to the Middle Resolution fibre-fed Echelle Spectrograph (MRES). The resulting spectra covered the 3900-8800 wavelength range at a resolving power of 15000 . Arc spectra were taken at the beginning of each night and we used the sky lines to account for the flexure of the spectrograph. 
Table 3. The radial velocities of the 1453 LAMOST WD+FGK binary candidates. The Heliocentric Julian Dates (HJD) and telescopes used for obtaining the spectra are also listed. ' -9999 ' indicates that no radial velocity is available. The complete table can be found in the electronic version of the paper.

\begin{tabular}{lcccc}
\hline Name & $\begin{array}{c}\text { HJD } \\
(\mathrm{d})\end{array}$ & $\begin{array}{c}\text { RV } \\
\left(\mathrm{km} \mathrm{s}^{-1}\right)\end{array}$ & $\begin{array}{c}\text { Err } \\
\left(\mathrm{km} \mathrm{s}^{-1}\right)\end{array}$ & Telescope \\
\hline J000230.57+155618.1 & 2456199.15371 & -24.45 & 11.64 & LAMOST \\
J000230.57+155618.1 & 2456199.16547 & -16.71 & 13.52 & LAMOST \\
J000232.64+074305.5 & 2457309.07594 & -9999 & -9999 & LAMOST \\
J000232.64+074305.5 & 2457309.09912 & -9999 & -9999 & LAMOST \\
J000232.64+074305.5 & 2457309.12228 & -9999 & -9999 & LAMOST \\
J000243.58+270214.7 & 2456647.98403 & -23.47 & 11.62 & LAMOST \\
J000243.58+270214.7 & 2456647.99553 & -29.21 & 10.97 & LAMOST \\
J000243.58+270214.7 & 2456648.00691 & -23.53 & 10.70 & LAMOST \\
J000324.28+063732.3 & 2456618.03752 & 88.30 & 10.26 & LAMOST \\
J000324.28+063732.3 & 2456618.04911 & 85.30 & 10.29 & LAMOST \\
\hline
\end{tabular}

\subsection{San Pedro Mártir 2.12 m Telescope}

Follow-up spectroscopy of $5 \mathrm{WD}+\mathrm{FGK}$ binaries was obtained during a run in 2015 December 5-7 (see Table 2) using the Echelle spectrograph attached to the $2.12 \mathrm{~m}$ telescope at San Pedro Mártir Observatory in Baja California, México. We used a slit width of 2.8 arcsec, resulting in 3650-7300 $\AA$ wavelength coverage at a resolving power $R \sim 20000$. Arc spectra were obtained before and after each object.

\subsection{Telescopio Nazionale Galileo}

Four nights of observations were carried out at the Telescopio Nazionale Galileo in 2017 June (see Table 2), providing followup spectroscopy of 45 additional WD+FGK binaries. We used the High Accuracy Radial velocity Planet Searcher - North (HARPS$\mathrm{N})$ spectrograph equipped and the 1 arcsec fibre, resulting in the wavelength coverage of $\sim 3800-6900 \AA$. The resolving power of HARPS-N is 115000 . We used a second fibre to obtain arc lamp spectra that were then used to establish a generic pixel-wavelength relation.

\section{RADIAL VELOCITIES}

Including all LAMOST DR 4, SDSS DR 12 and our own followup spectroscopy we have at hand a total of 5885 spectra for 1453 WD+FGK binary candidates. We made use of these spectra to measure the radial velocities of our WD+FGK binaries as follows (the radial velocities are provided in Table 3 ).

We followed the method described by Rebassa-Mansergas et al. (2008) and Ren et al. (2013) to fit the Na I doublet at 5800 ̊ arising from all spectra obtained from our INT, SPM, CFHT and TNT observations (Table 2). To that end we used a combination of a second order polynomial and a double-Gaussian profile.

We used the same approach as above described for measuring the radial velocities from the LAMOST and SDSS spectra. However, we noted that in most cases the $\mathrm{Na}$ I doublet profile was poorly sampled thus resulting in radial velocities associated with large uncertainties. We hence searched for common, clean and sufficiently resolved atmospheric features typical of $\mathrm{F}, \mathrm{G}$ and $\mathrm{K}$ stars (we remind the reader that the WD features are not visible in the optical spectra of WD+FGK binaries) and found that the Ca II triplet at $\sim 8500 \AA$ was the most frequently visible, cleanest and strongest. We hence measured the LAMOST and SDSS radial velocities fitting the Ca II
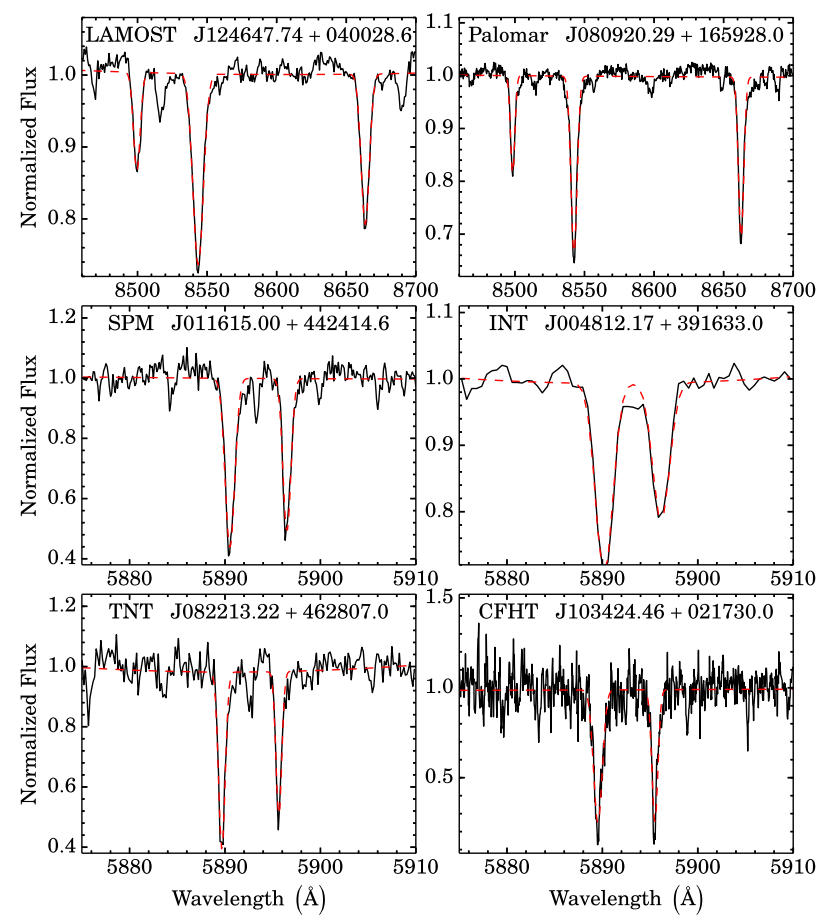

Figure 2. Fits (red dashed lines) to the Ca II triplet at $\sim 8500 \AA$ and the Na I doublet at $\sim 5800 \AA$ (black solid lines) for measuring the radial velocities. The telescopes used and object names are indicated in each panel.

absorption triplet with a combination of a second order polynomial and a triple-Gaussian profile of fixed separations. We used the same approach to measure the radial velocities from the red-arm spectra of our Palomar data since the blue-arm spectra covering other features such as the Na I doublet at $\sim 5800 \AA$ were not sufficiently resolved due to the low resolving power $(R=1380$, see Section 4.2). A few examples of double and triple Gaussian fits to the Ca II and $\mathrm{NaI}$ profiles are shown in Fig. 2.

Finally, we used the HARPS-N pipeline (Lovis \& Pepe 2007) that makes use of the cross-correlation function (Queloz 1995) to derive the radial velocities from the spectra obtained from our TNG observations.

\section{IDENTIFICATION OF CLOSE WD+FGK BINARIES}

We identified close WD+FGK binaries via a radial velocity analysis based on multi-epoch spectroscopy. That is, we considered a system to be a close binary if we detected significant $(>3 \sigma)$ radial velocity variation. To that end we combined all available radial velocities for each object, independently on the resolution of the spectra from which they were measured. ${ }^{3}$ However, it is important to keep in mind that we measured the radial velocities adopting three different methods depending on the data (Section 5), i.e. fitting the Ca II triplet at $\sim 8500 \AA$, fitting the $\mathrm{Na}$ I doublet at $\sim 5800 \AA$ or applying the cross-correlation function (see Section 5). Whilst both

\footnotetext{
${ }^{3}$ The time-scale in which we detect radial velocity variations varies from system to system, ranging from $1 \mathrm{~d}$ to $\sim 3 \mathrm{yr}$. The reason for this is twofold. First, observations were done in both visitor and service mode (with service mode observations obviously spanning a wider time range) and secondly we observed some systems with the same telescope (e.g. TNT and INT) in different years.
} 


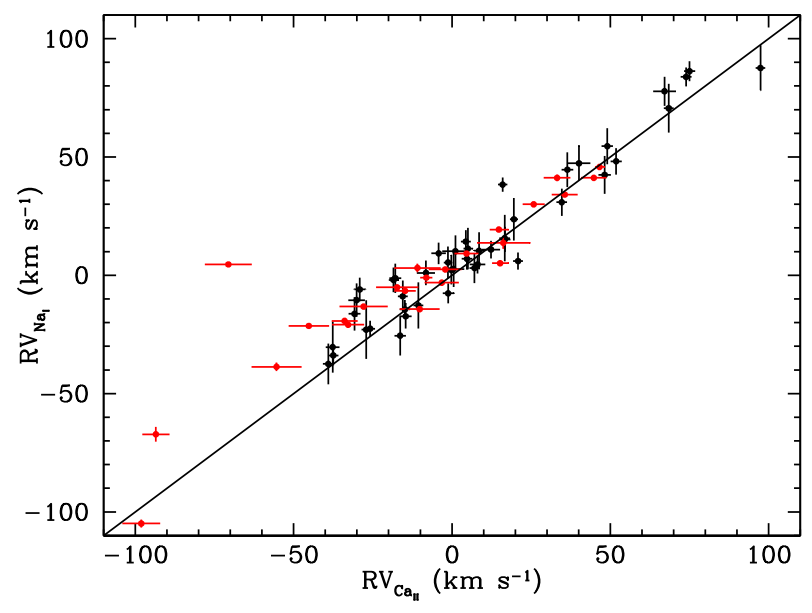

Figure 3. Comparison of the radial velocities measured from the Na I absorption doublet at $\sim 5800 \AA$ and from the Ca II absorption triplet at $\sim 8500 \AA$ of the same low-resolution LAMOST (black) and high-resolution CFHT (red) spectra of 45 and $24 \mathrm{WD}+$ FGK binaries, respectively.

the cross-correlation function and the Ca II triplet fitting technique have been proven to yield precise radial velocities - see Queloz (1995) and Kunder et al. (2017), respectively - the Na I doublet profile at $\sim 5800$ may be affected by interstellar absorption (Welsh et al. 2010). In order to explore possible effects of the interstellar absorption on the measurement of $\mathrm{Na}$ I radial velocities, we compare in Fig. 3 the $\mathrm{Ca}$ II absorption triplet and the $\mathrm{Na} \mathrm{I}$ absorption doublet radial velocities measured from 45 LAMOST high signal-to-noise ratio spectra $(>300)^{4}$ and 24 CFHT spectra of unique objects. It becomes obvious by looking at the figure that the radial velocities are in relatively good agreement, although discrepancies arise for some systems. Hence, in cases where a given WD+FGK binary has available radial velocities measured from both line profiles, we decide to not to combine them in order to search for radial velocity variations. Moreover, we only consider those values with uncertainties below $20 \mathrm{~km} \mathrm{~s}^{-1}$. This exercise results in 24 systems displaying more than $3 \sigma$ radial velocity variation that we classify as close binaries. The radial velocities of the 24 objects are displayed in Fig. 4, where we also include the objects names. It is worth mentioning that one of our identified close binaries is classified as an RS CVn binary by Simbad (J033336.49+384144.1, with an orbital period of $2.85 \mathrm{~d}$; Drake et al. 2014).

\section{DISCUSSION}

In the previous section, we identified $24 \mathrm{WD}+\mathrm{FGK}$ binaries displaying significant radial velocity variations. The number of systems with at least two available radial velocities that do not display radial velocity variation is 1402 , which, at face value, yields a close binary fraction of $\sim 1.5$ per cent. However, this value is misleading due to the following reasons.

First, in $\sim 70$ per cent of the cases the available radial velocities are obtained during the same night, which decreases considerably the probability of detecting close binaries with orbital periods longer than $1 \mathrm{~d}$. If we consider only WD+FGK systems with available radial velocities measured from spectra taken separated by at least

\footnotetext{
${ }^{4}$ The Na I doublet is poorly sampled by the low-resolution LAMOST spectra, hence we require high signal-to-noise ratio for reducing the radial velocity uncertainties.
}

one night, then the number of systems displaying and not displaying more than $3 \sigma$ radial velocity variations are 24 and 395 , respectively, i.e. a revised close binary fraction of $\sim 6$ per cent.

Secondly, low-inclination systems and systems with long orbital periods are harder to identify. This is not only because the detection probability decreases (see e.g. Nebot Gómez-Morán et al. 2011) but also because the radial velocity amplitudes of such systems are generally lower, hence higher resolution spectra are needed to derive radial velocities with lower uncertainties suitable for detecting small variations. This effect is important here since we used a wide variety of telescopes and instruments of resolving powers ranging from 1800 (LAMOST) to 115000 (TNG/HARPS). In order to quantify this effect, we consider a binary formed by a WD of $0.5 \mathrm{M}_{\odot}-$ a typical value for WDs in close WD plus main sequence binaries that evolved through mass transfer episodes, see Rebassa-Mansergas et al. (2011) - and a secondary star mass of $0.9 \mathrm{M}_{\odot}$ (which corresponds to a spectral type of $\sim \mathrm{G} 5$, the median value in our sample). Assuming orbital inclinations of 5, 20, 45 and $70 \mathrm{deg}$, we calculate the semi-amplitude velocities of the mainsequence stars employing the third law of Kepler for orbital periods up to $10000 \mathrm{~d}$. This is illustrated in the top panels of Fig. 5 (black solid curves). We then consider the $3 \sigma$ radial velocity variations that each combination of telescope/instrument used in this work is able to detect. We do this assuming a radial velocity error of 10 per cent the spectral resolution. The results are shown in the top panels of Fig. 5 as dashed horizontal lines. Inspecting the figures, it can be clearly seen that the LAMOST data are inefficient at detecting close binaries with orbital inclinations below $20 \mathrm{deg}$, but that close binaries with higher inclinations should be detected up to orbital periods of $\sim 3 \mathrm{~d}$ for an inclination of $45 \mathrm{deg}$ and $\sim 6 \mathrm{~d}$ for an inclination of $70 \mathrm{deg}$ (assuming that the spectra are obtained sufficiently separated to avoid sampling similar orbital phases, see the next paragraph). The orbital period limits increase for the INT and Palomar data, where we expect to be able to identify all close WD+FGK binaries with orbital periods up to $\sim 8.5 \mathrm{~d}$ and orbital inclinations higher than $70 \mathrm{deg}$. From our higher resolution spectra we expect be able to identify all close WD+FGK binaries with orbital periods up to $100 \mathrm{~d}$ (CFHT) and $300 \mathrm{~d}$ (TNG) at orbital inclinations $\gtrsim 5 \mathrm{deg}$ (again assuming that the spectra are taking at different orbital phases). These orbital period limits rely entirely on the assumption of a $0.5 \mathrm{M}_{\odot} \mathrm{WD}$ and a $0.9 \mathrm{M}_{\odot}$ main-sequence star (the most likely binary pair to be found among our sample of WD+FGK binaries), but of course many other combinations are possible. For completeness, we show two more examples in Fig. 5, middle $\left(0.6 \mathrm{M}_{\odot} \mathrm{WD}\right.$ and a $0.6 \mathrm{M}_{\odot}$ main-sequence star $)$ and bottom $\left(0.5 \mathrm{M}_{\odot} \mathrm{WD}\right.$ and a $1.2 \mathrm{M}_{\odot}$ main-sequence star) panels. One can see that, although the orbital period limits change from one case to another, the overall results are unchanged, i.e. the higher resolution data are more suitable to detect longer orbital period and/or lower inclination systems. Thus, if we only take into account WD+FGK systems with available radial velocities separated by more than one night and observed by the CFHT and TNG telescopes (our higher resolution data), the number of systems displaying and not displaying radial velocity variations is 6 and 58, i.e. a close binary fraction of $\sim 10$ per cent.

Thirdly, we need to take into account that the radial velocity values of a given system may be taken at the same orbital phase, which implies that some objects we catalogue as WD+FGK binaries that did not interact in their past are in fact close binaries. Moreover, it is important to keep in mind that, although our methodology for selecting WD+FGK systems based on GALEX colours is efficient - see Section 3 - the probability exists that we are selecting other 


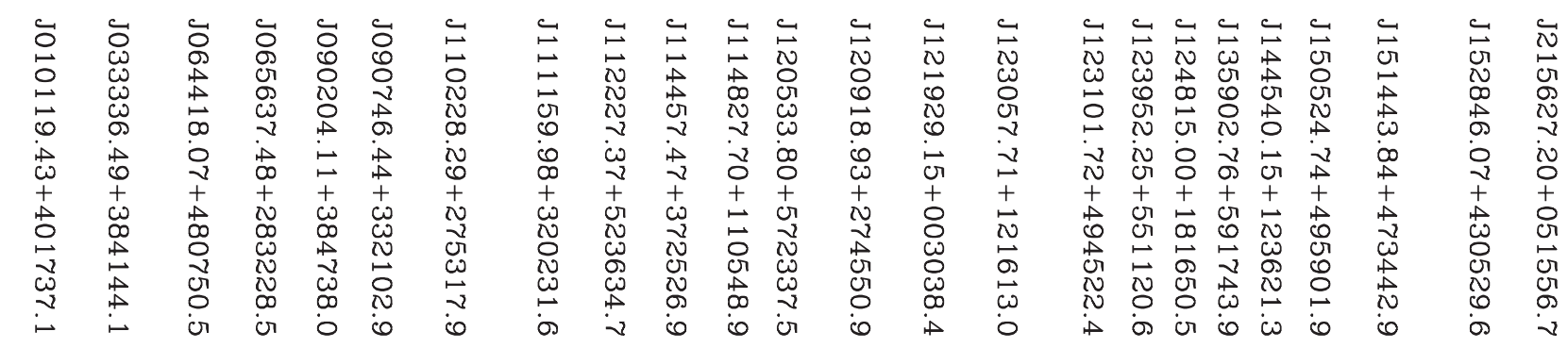

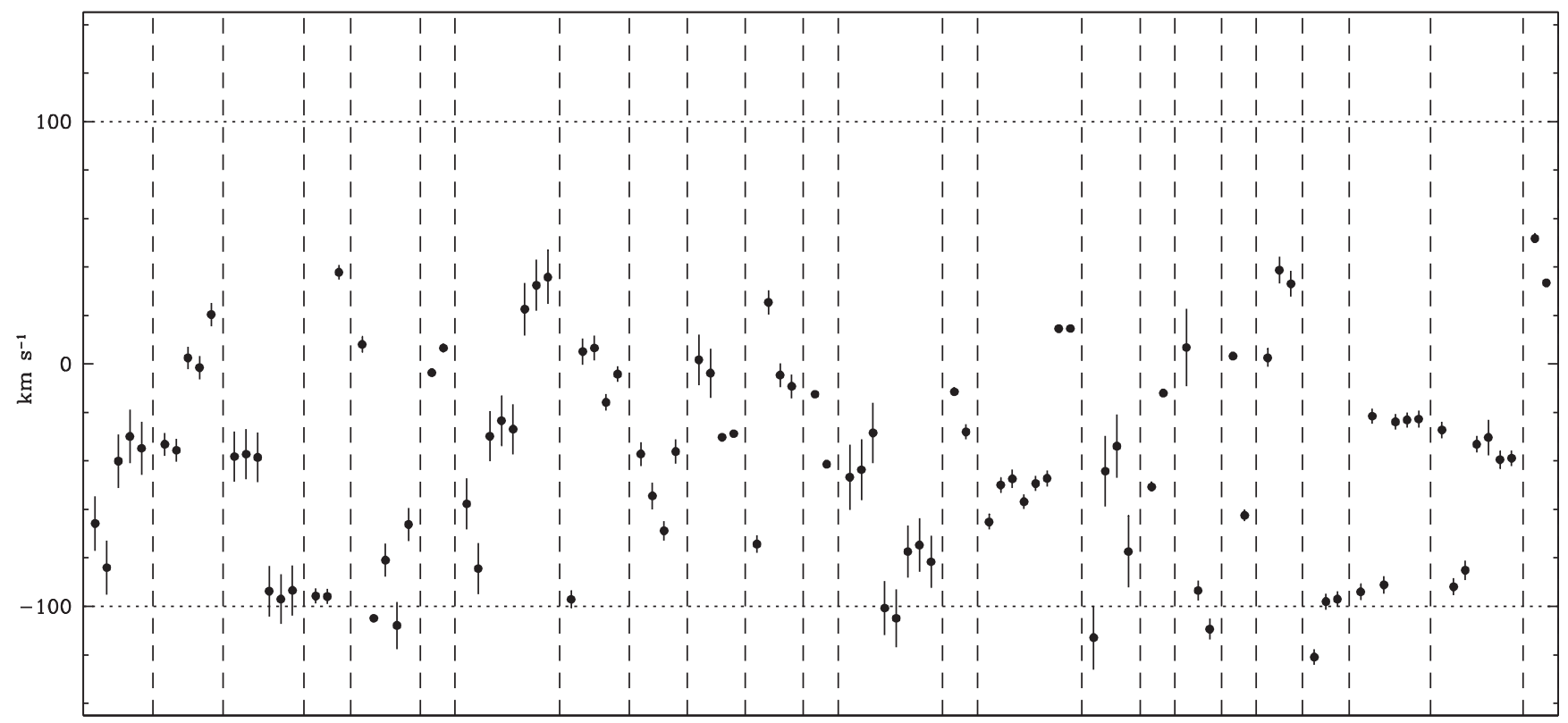

Figure 4. The radial velocities measured for the $24 \mathrm{WD}+\mathrm{FGK}$ binaries that display significant $(>3 \sigma)$ radial velocity variation. The object names are provided in the top.

kind of astronomical objects such as e.g. active main-sequence stars (Smith, Bianchi \& Shiao 2014) or RR Lyrae (Section 3). Quantifying the contamination from other sources in our sample requires UV spectra of a large number of sources to visually test the presence of the WD. The fact that we clearly observed the WD features in the Hubble Space Telescope of nine objects in our sample seems to indicate that the contamination should be small (Parsons et al. 2016).

Because of these reasons, the close binary fraction of $\sim 10$ per cent should be taken as preliminary. This value is considerably lower than the close binary fraction resulting from our analysis of WDs in orbits with $\mathrm{M}$ dwarf companions (WD+M binaries) ( $\sim 25$ per cent; Nebot Gómez-Morán et al. 2011). This is likely because the $\mathrm{WD}+\mathrm{M}$ sample contains fully convective stars for which magnetic braking is expected to be largely reduced (Reiners \& Basri 2009; Morin et al. 2010). Hence, angular momentum loss is much more inefficient at bringing these (WD+M) binary components together for initiating a semidetached phase (Politano \& Weiler 2006; Schreiber et al. 2010; Rebassa-Mansergas, Schreiber \& Gänsicke 2013; Zorotovic et al. 2016). In other words, we expect a larger fraction of WD+FGK binaries to have become semidetached than WD+M systems, in line with the observed close binary fractions. An additional possibility may be that close WD+FGK systems emerge from common envelope at longer periods (weeks/months) than WD+M systems, hence they are harder to identify (especially by our lower resolution data). This would imply a higher common envelope efficiency for the WD+FGK systems. Indeed, this seems to be the case for the two long-orbital period WD+FGK binaries studied by Zorotovic, Schreiber \& Parsons (2014).

\section{CONCLUSIONS}

With the aim of testing the importance of the single- and doubledegenerate channels for Type Ia supernovae (SNIa), we have started an observing campaign dedicated to identify detached close WD+FGK binaries and to measure their orbital periods and component masses. With these data at hand, we will be able to predict what systems will initiate a phase of thermal-time-scale mass transfer and thus become super-soft sources and potential singledegenerate SN Ia progenitors, and what binaries will evolve through a second common envelope phase and form double white dwarfs, i.e. potential double-degenerate SN Ia progenitors. In this paper, the second of a series of publications, we identified 1,549 WD+FGK binary candidates from the DR4 of LAMOST and presented followup spectroscopy of 1453 of them. Analysing the radial velocities, we have detected 24 close binaries displaying significant radial velocity variation and calculated a preliminary close binary fraction among WD+FGK systems of $\sim 10$ per cent. We have also shown that high-resolution spectra are needed to efficiently identify lower inclination ( $\lesssim 5 \mathrm{deg}$ ) and/or longer orbital period systems ( $\gtrsim 100 \mathrm{~d}$; in these cases service mode observations are additionally required). This is important for detecting WD+FGK systems that will evolve through a second common envelope phase and avoid merging to thus become double-degenerate SN Ia progenitors candidates. In a forthcoming publication, we will present additional follow-up observations of WD+FGK binaries identified by the RAVE survey in the Southern hemisphere as well as our first orbital periods measured. 

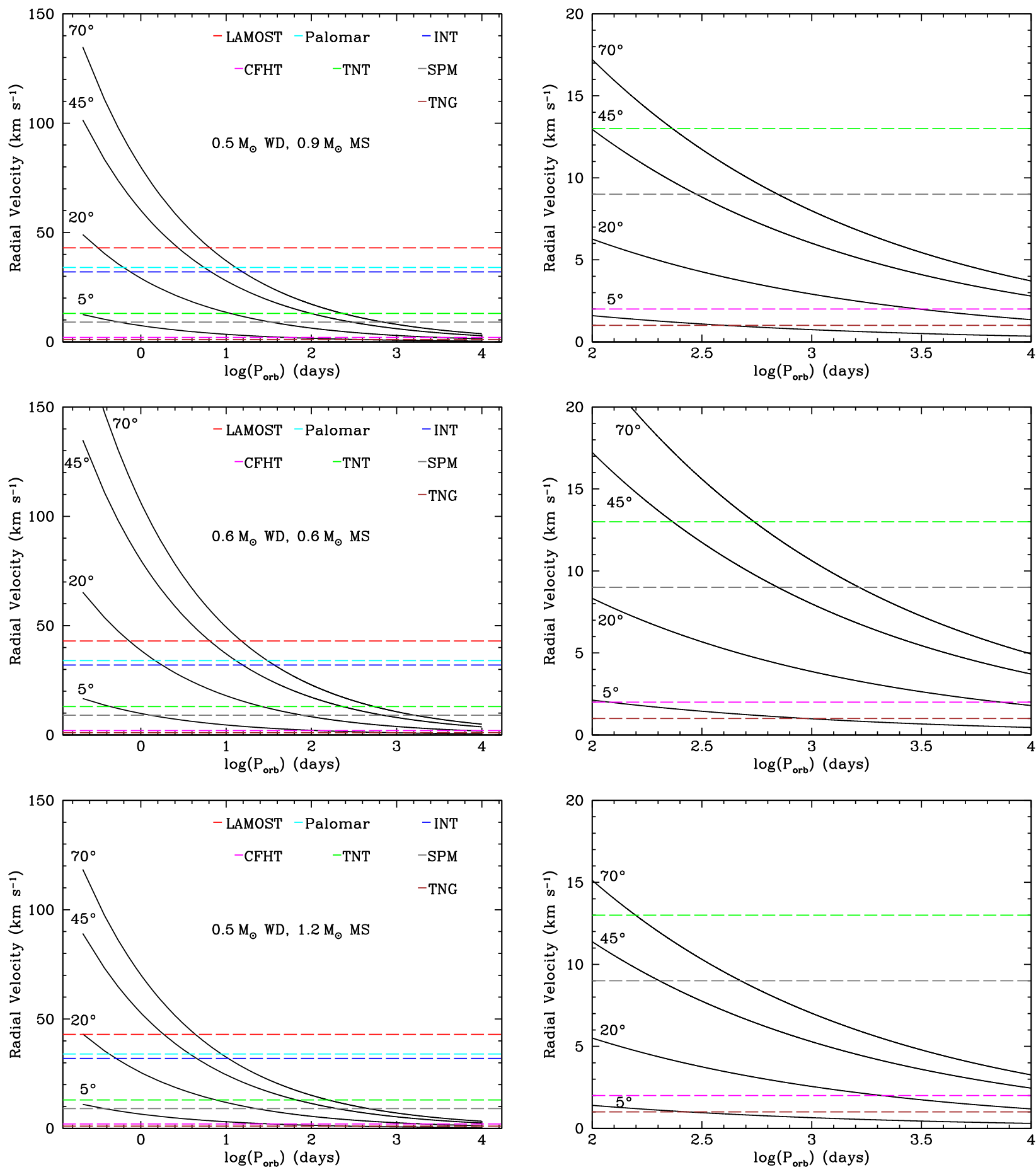

Figure 5. In black solid lines, we represent the semi-amplitude radial velocities of the main-sequence stars in WD+FGK binaries formed by a $0.5 \mathrm{M}_{\odot}$ WD and a $0.9 \mathrm{M}_{\odot}$ main-sequence star (top panels), a $0.6 \mathrm{M}_{\odot} \mathrm{WD}$ and a $0.6 \mathrm{M}_{\odot}$ main-sequence star (middle panels) and a $0.5 \mathrm{M}_{\odot} \mathrm{WD}$ and a $1.2 \mathrm{M}_{\odot} \mathrm{main}$ sequence (bottom panels) as a function of orbital period for the assumed inclinations of 5, 20, 45 and 70 deg. The right-hand panels are simply a zoom in to the longer orbital periods considered. The horizontal dashed lines indicate the $3 \sigma$ radial velocity variation that the telescopes/instruments used in this work can detect. As expected, the $3 \sigma$ radial velocity variation decreases for increasing values of the spectral resolution.

\section{ACKNOWLEDGEMENTS}

This research has been funded by the Agència de Gestió d'Ajuts Universitaris i de Recerca, by Agència de Gestió d'Ajuts Universitaris i de Recerca grants AYA2014-59084-P (ARM, EGB) and AYA2014-55840-P (EV), by the Young Researcher Grant of National Astronomical Observatories, Chinese Academy of Sci- ences (JJR), by the Key Basic Research Program of China (XL; 2014CB845700), by the Leverhulme Trust (SGP), by the European Research Council under the European Union's Seventh Framework Programme (BTG; FP/2007-2013)/ERC grant agreement n.320964 (WDTracer) and by Fondecyt (MRS; grant 1141269).

This work has made use of data products from the Guoshoujing Telescope (the Large Sky Area Multi-Object Fibre Spectroscopic 
Telescope, LAMOST). LAMOST is a National Major Scientific Project built by the Chinese Academy of Sciences. Funding for the project has been provided by the National Development and Reform Commission. LAMOST is operated and managed by the National Astronomical Observatories, Chinese Academy of Sciences.

Based on observations performed at the Palomar Hale Telescope (time obtained through Chinese Telescope Access Program, 2013B20), the Isaac Newton Telescope (programs 2014B-80, 2015B-7, 2016B-33), the Canada France Hawaii Telescope (time obtained through Chinese Telescope Access Program, 2015B-27), the Thai National Telescope, the 2.12 m telescope in San Pedro Mártir Observatory and the Telescopio Nazionale Galileo (program CAT17A-5).

\section{REFERENCES}

Astier P., Pain R., 2012, C. R. Phys., 13, 521

Aznar-Siguán G., García-Berro E., Lorén-Aguilar P., José J., Isern J., 2013, MNRAS, 434, 2539

Bours M. C. P., Toonen S., Nelemans G., 2013, A\&A, 552, A24

Carlin J. L. et al., 2012, Res. Astron. Astrophys., 12, 755

Chen L. et al., 2012, Res. Astron. Astrophys., 12, 805

Cui X.-Q. et al., 2012, Res. Astron. Astrophys., 12, 1197

Deng L.-C. et al., 2012, Res. Astron. Astrophys., 12, 735

Di Stefano R., 2010, ApJ, 712, 728

Drake A. J. et al., 2009, ApJ, 696, 870

Drake A. J. et al., 2014, ApJS, 213, 9

Fink M., Hillebrandt W., Röpke F. K., 2007, A\&A, 476, 1133

Fujimoto M. Y., 1982, ApJ, 257, 752

Gal-Yam A., Maoz D., 2004, MNRAS, 347, 942

Gänsicke B. T. et al., 2003, ApJ, 594, 443

García-Berro E. et al., 2012, ApJ, 749, 25

García-Berro E., Soker N., Althaus L. G., Ribas I., Morales J. C., 2016, New A, 45,7

González Hernández J. I., Ruiz-Lapuente P., Tabernero H. M., Montes D., Canal R., Méndez J., Bedin L. R., 2012, Nature, 489, 533

Hachisu I., Kato M., Saio H., Nomoto K., 2012, ApJ, 744, 69

Hamers A. S., Pols O. R., Claeys J. S. W., Nelemans G., 2013, MNRAS, 430, 2262

Hamuy M. et al., 2003, Nature, 424, 651

Han Z., Podsiadlowski P., 2004, MNRAS, 350, 1301

Henden A. A., Levine S., Terrell D., Welch D. L., 2015, Am. Astron. Soc. Meeting Abstr. 225, 336.16

Howell D. A., 2011, Nature Commun., 2, 350

Hoyle F., Fowler W. A., 1960, ApJ, 132, 565

Hussain G. A. J., Allende Prieto C., Saar S. H., Still M., 2006, MNRAS, 367,1699

Iben I. J., 1982, ApJ, 259, 244

Iben I., Jr, Tutukov A. V., 1984, ApJS, 54, 335

Idan I., Shaviv N. J., Shaviv G., 2012, J. Phys. Conf. Ser., 337, 012051

Jiménez N., Tissera P. B., Matteucci F., 2015, ApJ, 810, 137

Kashi A., Soker N., 2011, MNRAS, 417, 1466

Katz B., Dong S., Malhotra R., 2011, Phys. Rev. Lett., 107, 181101

Kordopatis G. et al., 2013, AJ, 146, 134

Kunder A. et al., 2017, AJ, 153, 75

Kushnir D., Katz B., Dong S., Livne E., Fernández R., 2013, ApJ, 778, L37

Linden S., Virey J.-M., Tilquin A., 2009, A\&A, 506, 1095

Liu Z.-W., Stancliffe R. J., 2016, MNRAS, 459, 1781

Liu X.-W. et al., 2014, in Feltzing S., Zhao G., Walton N. A., Whitelock P., eds., Proc. IAU Symp. 298, Setting the scene for Gaia and LAMOST. Lijiang, China, p. 310

Livio M., Riess A. G., 2003, ApJ, 594, L93

Livne E., Arnett D., 1995, ApJ, 452, 62

Lovis C., Pepe F., 2007, A\&A, 468, 1115

Luo A.-L. et al., 2012, Res. Astron. Astrophys., 12, 1243

Luo A.-L. et al., 2015, Res. Astron. Astrophys., 15, 1095

Maoz D., Badenes C., 2010, MNRAS, 407, 1314
Maoz D., Sharon K., Gal-Yam A., 2010, ApJ, 722, 1879

Martin D. C. et al., 2005, ApJ, 619, L1

Morin J., Donati J.-F., Petit P., Delfosse X., Forveille T., Jardine M. M., 2010, MNRAS, 407, 2269

Nebot Gómez-Morán A. et al., 2011, A\&A, 536, A43

Nielsen M. T. B., Nelemans G., Voss R., Toonen S., 2014, A\&A, 563, A16

Nomoto K., 1982, ApJ, 253, 798

Nomoto K., Iben, Jr I., 1985, ApJ, 297, 531

Olling R. P. et al., 2015, Nature, 521, 332

Parsons S. G. et al., 2015a, MNRAS, 449, 2194

Parsons S. G. et al., 2015b, MNRAS, 452, 1754

Parsons S. G., Rebassa-Mansergas A., Schreiber M. R., Gänsicke B. T., Zorotovic M., Ren J. J., 2016, MNRAS, 463, 2125

Perlmutter S. et al., 1999, ApJ, 517, 565

Phillips M. M., 1993, ApJ, 413, L105

Politano M., Weiler K. P., 2006, ApJ, 641, L137

Queloz D., 1995, in Philip A. G. D., Janes K., Upgren A. R., eds., Proc. IAU Symp. 167, New Developments in Array Technology and Applications. The Netherland, p. 221

Rebassa-Mansergas A. et al., 2008, MNRAS, 390, 1635

Rebassa-Mansergas A., Gänsicke B. T., Schreiber M. R., Koester D., Rodríguez-Gil P., 2010, MNRAS, 402, 620

Rebassa-Mansergas A., Nebot Gómez-Morán A., Schreiber M. R., Girven J., Gänsicke B. T., 2011, MNRAS, 413, 1121

Rebassa-Mansergas A., Schreiber M. R., Gänsicke B. T., 2013, MNRAS, 429, 3570

Rebassa-Mansergas A., Parsons S. G., Copperwheat C. M., Justham S., Gänsicke B. T., Schreiber M. R., Marsh T. R., Dhillon V. S., 2014, ApJ, 790,28

Reiners A., Basri G., 2009, A\&A, 496, 787

Ren J., Luo A., Li Y., Wei P., Zhao J., Zhao Y., Song Y., Zhao G., 2013, AJ, 146,82

Riess A. G. et al., 1998, AJ, 116, 1009

Santander-García M., Rodríguez-Gil P., Corradi R. L. M., Jones D., Miszalski B., Boffin H. M. J., Rubio-Díez M. M., Kotze M. M., 2015, Nature, 519,63

Schenker K., King A. R., Kolb U., Wynn G. A., Zhang Z., 2002, MNRAS, 337,1105

Schmidt B. P., Suntzeff N. B., Phillips M. M. et al., 1998, ApJ, 507, 46

Schreiber M. R. et al., 2010, A\&A, 513, L7+

Shappee B. J. et al., 2016, ApJ, 826, 144

Shara M. M., Prialnik D., Shaviv G., 1977, A\&A, 61, 363

Shen K. J., Bildsten L., Kasen D., Quataert E., 2012, ApJ, 748, 35

Smith M. A., Bianchi L., Shiao B., 2014, AJ, 147, 159

Soker N., Kashi A., García-Berro E., Torres S., Camacho J., 2013, MNRAS, 431,1541

Soker N., García-Berro E., Althaus L. G., 2014, MNRAS, 437, L66

Thompson T. A., 2011, ApJ, 741, 82

Thorstensen J. R., Fenton W. H., Patterson J., Kemp J., Halpern J., Baraffe I., 2002, PASP, 114, 1117

Totani T., Morokuma T., Oda T., Doi M., Yasuda N., 2008, PASJ, 60, 1327

van den Heuvel E. P. J., Bhattacharya D., Nomoto K., Rappaport S. A., 1992, A\&A, 262, 97

van Rossum D. R., Kashyap R., Fisher R., Wollaeger R. T., García-Berro E., Aznar-Siguán G., Ji S., Lorén-Aguilar P., 2016, ApJ, 827, 128

Voss R., Nelemans G., 2008, Nature, 451, 802

Wang B., Han Z., 2012, New A Rev., 56, 122

Welsh B. Y., Lallement R., Vergely J.-L., Raimond S., 2010, A\&A, 510, A54

Whelan J., Iben I., Jr, 1973, ApJ, 186, 1007

Willems B., Kolb U., 2004, A\&A, 419, 1057

Wolf W. M., Bildsten L., Brooks J., Paxton B., 2013, ApJ, 777, 136

Woosley S. E., Weaver T. A., 1994, ApJ, 423, 371

Wu Y., Singh H. P., Prugniel P., Gupta R., Koleva M., 2011, A\&A, 525, A71

Xiang M.-S. et al., 2017a, MNRAS, 464, 3657

Xiang M.-S. et al., 2017b, MNRAS, 467, 1890

York D. G. et al., 2000, AJ, 120, 1579

Yuan H.-B. et al., 2015, MNRAS, 448, 855 
Yungelson L. R., Kuranov A. G., 2017, MNRAS, 464, 1607

Zhang H.-H., Liu X.-W., Yuan H.-B., Zhao H.-B., Yao J.-S., Zhang H.-W., Xiang M.-S., Huang Y., 2014, Res. Astron. Astrophys., 14, 456

Zhao G., Zhao Y.-H., Chu Y.-Q., Jing Y.-P., Deng L.-C., 2012, Res. Astron. Astrophys., 12, 723

Zorotovic M., Schreiber M. R., Gänsicke B. T., 2011, A\&A, 536, A42

Zorotovic M., Schreiber M. R., Parsons S. G., 2014, A\&A, 568, L9

Zorotovic M. et al., 2016, MNRAS, 457, 3867

\section{SUPPORTING INFORMATION}

Supplementary data are available at MNRAS online.

Table 1. The selected 2168 spectra of 1549 unique LAMOST DR 4 WD+FGK binary candidates (see Fig. 1), including coordinates, GALEX UV magnitudes, spectral ID identifiers (plate, spectrograph, fibre IDs and modified Julian date MJD), signal-to-noise ratio in different spectral bands and stellar parameters. The 'Mag' column provides the optical magnitudes obtained from AAVSO Photometric All Sky Survey DR9 (APASS; Henden et al. 2015), although some entries are obtained from the Catalina Sky Survey (CSS; Drake et al. 2009) or Guiding Star Catalog (GSC).

Table 3. The radial velocities of the 1453 LAMOST WD+FGK binary candidates.

Please note: Oxford University Press is not responsible for the content or functionality of any supporting materials supplied by the authors. Any queries (other than missing material) should be directed to the corresponding author for the article.

This paper has been typeset from a $\mathrm{T}_{\mathrm{E}} \mathrm{X} / \mathrm{L} \mathrm{T}_{\mathrm{E}} \mathrm{X}$ file prepared by the author. 